\title{
Biological Psychiatry and Reductionism Empirical Findings and Philosophy
}

\author{
HASSE KARLSSON and MATTI KAMPPINEN
}

The main perspective in psychiatry has shifted during recent years from the psychodynamic to the biological. In this paper it is argued, firstly - in the line of Rudnick (1990), Callaway (1992), Freeman (1992), Mass \& Katz (1992) and Mandell \& Selz (1992) - that biological psychiatry is in a confused state; secondly, that this confusion is due in part to the difficulty of interpreting new neurobiological data because of a lack of a sound conceptual framework. Thirdly, we argue that these confusions are related to the issue of levels, in that the levels of organisation are not explicated with sufficient clarity in biological psychiatry. This problem of levels in psychiatry is well expressed in the comment that psychiatry of the past was brainless, but that psychiatry of the future seems to be mindless (Eisenberg, 1986).

In the following we discuss the concept of reduction. We propose that emergent materialism is a plausible stance in biological psychiatry and that it clarifies the concepts of reduction and level in psychiatry.

\section{Reduction in biological psychiatry}

The basic assumption in biological research is that of reductionism. In biological psychiatry too, the simplest models are built upon the principle of reduction. Often biological psychiatry has been condemned as mechanistic and simplistic (Pam, 1990). We should, however, take a closer look at what precisely is meant by the notion of reduction (Bunge, 1977; Churchland, 1982, 1986).

The most obvious and popular case of reduction is theory reduction, which can be characterised as a process whereby a theory is reduced to another, more basic theory. The reduction may be complete, in the sense that it shows that the old theory was false, but sometimes the old theory is integrated into the new one. The natural sciences (physics, chemistry, biology) have usually been seen as the most basic theories. Psychology and the social sciences are commonly considered to be either basically non-existent or in any case secondary to natural science.

The strongest form of reductionism is the view that, while mental concepts may or may not be reducible to neurophysiological ones, the categories of psychology are in any case so vague that they should be eliminated from science altogether. According to this stance, sooner or later all psychological concepts will be replaceable with specific neurobiological concepts. This position is often referred to as eliminativism.

Despite their popularity in philosophy, very few empirical scientists endorse theory reduction or eliminativism. Insofar as these stances involve the idea of type-identity, existing empirical data can be interpreted as evidence against them. Type-identities would require that the mental and the physical types are one-to-one. That is, that the mental and the physical divisions of the brain are isomorphic. As pointed out in a recent paper by Volkow \& Tancredi (1991), positron-emission tomography (PET) findings have shown that there are differences among individuals in the strategies used to process a given stimulus. They conclude that trying to associate any particular brain area with a given mental operation is not feasible because mental operations are the result of coordinated activation of various brain areas. Furthermore, defects in different brain areas can generate the same type of mental dysfunction. According to Volkow \& Tancredi (1991), models which require a one-to-one relationship between the physical and mental are not plausible. A better model allows an association between clusters of physical processes and one or more mental phenomena.

A weaker version of the reductionist stance states that mental phenomena are caused by neurobiological events alone. This stance is called causal reductionism, and seems to be the position held by many biological psychiatrists. Although psychological phenomena exist, they are secondary. This resembles epiphenomenalism, according to which the mind is 'secreted' by the brain.

Causal reductionism implies causal effects only in a 'bottom-up' direction, from neurobiological events to psychological phenomena. The problem with this stance is that it has difficulty in explaining findings indicating that similar neurobiological effects may differ at the experiential or behavioural level according to context (Callaway, 1992). It can of course be argued 
that the neurobiological determinants of behaviour are too complex, but a more plausible possibility is that biological processes and their effects can be influenced by phenomena occurring at higher levels (psychological, social). Recent empirical findings (Pardo et al, 1993) which imply downstream causality are likewise not explicable in terms of causal reduction (we will come back to this notion below).

These reductionist models have been popular partly due to their simplicity. Their disadvantage, on the other hand, has been that they have fostered research designs in biological psychiatry which seem to favour over-simplistic interpretations of the results. The last few years, however, have witnessed a quest for non-reductionist models in psychiatry, and the emergence of new theories, whose basic characteristic is the absence of this tendency towards simple reductionism. In biological psychiatry too there have been comments in this direction (Callaway, 1992; Mandell \& Selz, 1992). All in all, the question of the relationship between biological and psychological events and concepts seems to be once more the subject of debate in psychiatry (Reiser, 1984; Pardes, 1986; Hundert, 1989; van Praag, 1989; Gabbard, 1992).

\section{Problems with reduction}

Reduction has two aspects: ontological and epistemological. Ontological reduction is characteristic of eliminativism. The qualities of a higher-level phenomenon are reduced to phenomena at a lower level. Epistemological reduction, on the other hand, strives to understand certain aspects of a phenomenon in terms of neurophysiological statements. It does not eliminate the qualitative variety of existence, as does ontological reduction (Bunge, 1980). Bunge (1977) offers an example of this difference. When water is equated with $\mathrm{H}_{2} \mathrm{O}$, this is an example of epistemological reduction. Along with its chemical composition, however, water also has a structure, which implies that water has certain emergent properties such as viscosity and fluidity.

Moreover, epistemological reduction can be more or less complete. For instance, in the recent discussion based on empirical studies, it has been said that the 'specific disease, specific biology' approach should be abandoned and replaced by a focus on the relationship between the functioning of neurotransmitter systems and the more fundamental components of mental disorders, such as emotions and the elements of cognition (Maas \& Katz, 1992). In the same line of reasoning are the findings of Hudson \& Pope (1990), who point out that in terms of a treatment-response model it is possible to identify eight disorders with a shared pathophysiologic abnormality (major depression, bulimia, panic disorder, obsessive-compulsive disorder, attention deficit disorder with hyperactivity, cataplexy, migraine and irritable bowel syndrome). If this is the case, then illness categories cannot be explained by reference solely to events on a single level. This may imply that complete epistemological reduction is not available in these cases.

It has even been argued recently that the goal of treatment is to induce global brain changes; these are achieved by seemingly illogical procedures (eg. reduction of anti-depressant medication in cases of treatment failure), but are understandable in terms of nonlinear systems (Mandell \& Selz, 1992). These ideas, according to the authors, may contribute to a new unity of the behavioural and brain sciences.

The whole idea of reduction is in contradiction with the notion of 'downstream causality' (Hofstadter, 1981; Sperry, 1980; Érdi, 1993; Scentágothai, 1993). Pardo et al (1993) have reported that, using the PET technique, they were able to measure changes in the regional cerebral blood flow of normal volunteers during self-induced dysphoria. Gabbard (1992) concludes that the human brain undergoes significant functional and anatomical change in response to psychological influences.

Some clinical studies also seem to support the notion that psychological and social processes act on biological phenomena in a more global sense. One example is the study by Spiegel et al (1989), who demonstrated that psychosocial treatment may increase the survival time of patients with metastatic breast cancer. Also Fawzy et al (1993) reported increased survival time after psychiatric intervention among patients with malignant melanoma. It has even been suggested that, on the basis of what is known about psychobiological interactions, psychotherapy should be considered a biological treatment (Mohl, 1987).

It can thus be concluded that the search for explanation needs to reach out to other levels besides the biological: the psychological and the social.

\section{Emergent materialism and biological psychiatry: a balance between solutions and additional problems}

Emergent materialism is a philosophical doctrine which renders many psychological and psychiatric findings intelligible. Before going into details, it is worth mentioning that emergent materialism also 
generates problems of its own, mainly because it offers solutions to the questions posed by $\mathrm{mind} / \mathrm{brain}$ interactions. The reader should thus weigh the number of problems answered against the number of new problems begotten. But first, what is emergent materialism?

According to Mario Bunge (1981), who can be considered its most outspoken proponent, emergent materialism is an ontological doctrine which states, flatly, that everything is material. More precisely, everything is either a material thing, a material system, a property of a material system, or an aspect dependent upon material systems. This accounts for the 'materialism' part of the doctrine.

Emergent materialism differs from other, more vulgar versions of materialism in that it allows for the apparent richness of existence by positing multiple levels of organisation. By the 'apparent richness of existence' we mean the commonsense fact that there appear to be several kinds of things in the world: physical middle-sized objects and their constituents, human beings and their organs, institutions made of human beings, and so on. A level of organisation is a collection of systems such that the components of these systems belong to a lower level (see Fig. 1).

Higher levels 'emerge' from lower ones. Emergence has two aspects: higher levels are dependent upon lower ones, but are at the same time lawful in their own right. This means that the laws of the higher level are not generally deducible from the laws of the lower one. Higher levels host novel, emergent properties, not found at the lower level.

'Supervenience' is a technical term used to describe the relationship of dependence between adjacent levels in the hierarchy. There are two kinds of supervenience: weak and strong. A higher-level system $X$ is weakly supervenient on a lower level system $Y$ if a change in $X$ requires a change in $Y$. $X$ is strongly supervenient on $Y$ if a change in $Y$ causes a change in $\mathrm{X}$. Most mental/physical relations are cases of weak supervenience. $A$ change in the neural system can take place without a corresponding change in the mental system.

Supervenience is description-relative: before supervenience can be located in the system under consideration, the system must be divided into levels. Take, for example, the brain. It appears obvious that psychological phenomena, such as 'thinking about summer' or 'being afraid of the dark' are dependent upon neural happenings. Whether the dependence in question involves weak or strong supervenience, depends somewhat on how neural activities are described.

Let us assume that the change at the psychological level is the transition from thinking about summer to

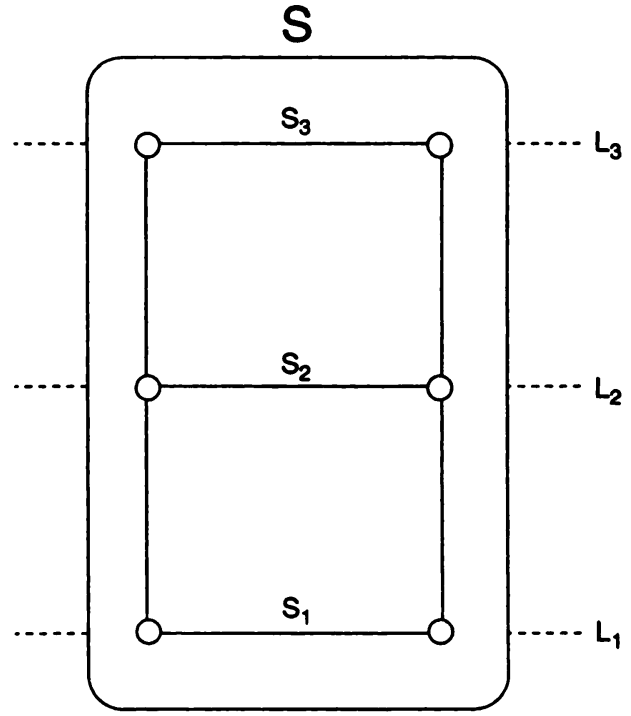

Fig. 1 The system $S$ exists in three different levels $L_{,}, L_{2}, L_{\text {, }}$. For example, a human being exists on biological $\left(L_{1}\right)$, psychological $\left(\mathrm{L}_{2}\right)$ and social $\left(\mathrm{L}_{3}\right)$ levels. The human being can be divided into level-specific subsystems $S_{1}, S_{2}, S_{3}$.

being afraid of the dark. In order for this to be a case of weak supervenience, we need to describe a level of neural happenings where, whenever such a psychological change occurs, a corresponding neural event also occurs. A description that could predict for example which part of the brain will have the greatest amount of activity going on would qualify as weak supervenience.

In order to qualify as strong supervenience, we would need a more detailed description, such that whenever the neural change appeared, so would the corresponding psychological change.

A widely accepted view is that in the case of the psychological/neural relationship, there is no way to construe a case of strong supervenience. On the basis of what has just been said, it can be argued that the validity of this view depends on the way the levels are described. If for example the psychological level involves a change from thinking about one's grandmother to thinking about the grandmother holding a glass of milk in her hand, there is no place to look for a sufficient neural change. If, on the other hand, the psychological level involves changes from thinking to being afraid, then the prospects are better.

All in all, since supervenience is descriptionrelative, the question whether a level $L_{1}$ is weakly or strongly supervenient on a level $L_{2}$ is partly an empirical question. Levels of being are identified $a$ posteriori, on the basis of empirical investigation. 
To sum up, emergent materialism is an attractive ontology for the following reasons: (1) it accounts for our intuitions that there are multiple levels of organisation in the world; (2) it provides a theory of how these levels are linked; (3) it offers an outline of the possible modes of lawfulness that can exist between levels.

The doctrine outlined is less attractive because there are no satisfactory theories of emergence or supervenience available, and labelling the inter-level relationships with new names merely serves to mystify them. (Recently, however, 'self-organisation' has been proposed to account for emergent properties in the neural system (Scentágothai, 1993). Spontaneous neural activity has been empirically demonstrated to result in 'biologically significant' behaviour (Érdi, 1993). This might provide a clue for building a future theory of emergence.)

The problem of emergence gives rise to another fundamental question, namely, the question of intelligibility: What counts as an explanation? Is anything complex ever explained, or do typewriters, for example, remain a mystery in relation to their components?

Thus we are back where we began: emergent materialism was introduced because it renders many psychiatric findings intelligible. To assess its worth, we must first find out what we mean by intelligibility. In psychiatry one criterion for intelligibility could be the clinical relevance or usefulness of a theory. Emergent materialism seems to fill a gap in recent psychiatric discussion. This gap concerns the relationship between events on different levels. At the same time, however, it opens up some new gaps, which will have to be filled in the future by empirical research and theory.

\section{Conclusions}

Some of the results of research in biological psychiatry are clinically problematic, because the research designs seem to favour the reductionist model. An example of what is meant by this is given in Dennett (1979), in his illuminating reinterpretation of the Skinner experiments. He shows that Skinner's experiment setting allows for interpretations of only a reductive sort. The research designs in biological psychiatry too have ontological commitments and implications concerning the interpretation of the results. Recent studies which cross the boundaries between biological, psychological and social levels simulate genuine clinical situations with greater accuracy. The results of these studies can be understood within the conceptual framework of emergent materialism.
Clinically, a biological psychiatry committed to a strong version of reduction is of limited use. This is nicely shown in the paper by Gabbard (1992). We cannot exclude effects stemming from other levels, i.e. psychological, interpersonal and social processes, which modify for instance the psychopharmacological outcome, even in the most 'biological' cases. The reduction of the clinical situation is partly acceptable only in research settings. This does not, however, mean that the results of psychopharmacological or other biological research in psychiatry are obscure. The main question is how to interpret and use these results.

\section{References}

Bunge, M. (1977) Emergence and the mind. Neuroscience, 2, 501-509.

- (1980) The Mind-Body Problem. A Psychobiological Approach. Oxford: Pergamon Press.

(1981) Scientific Materialism. Dordrecht: Reidel.

Callaway, E. (1992) Psychopharmacology's need for linking variables. Biological Psychiatry, 31, 1-3.

ChurChland, P. S. (1982) Mind-brain reduction: New light from the philosophy of science. Neuroscience, 7, 1041-2047.

(1986) Neurophilosophy: Toward a Unified Science of the Mind-brain. Cambridge: MIT Press.

Dennetr, D. (1979) Skinner skinned. In Brainstorms. Philosophical Essays on Mind and Psychology (ed. D. C. Dennett). Hassocks, Sussex: Harvester Press.

EISENBERG, L. (1986) Mindlessness and brainlessness in psychiatry. British Journal of Psychiatry, 148, 497-508.

ÉrDI, P. (1993) Neurodynamic system theory: Scope and limits. Theoretical Medicine, 14,137-152.

FAwzy, F. I., FAWzy, N. W., Hyun, C. S., et al (1993) Malignant melanoma. Effects of an early structured psychiatric intervention, coping and affective state on recurrence and survival 6 years later. Archives of General Psychiatry, 50, 681-689.

Freeman, W. (1992) Chaos in psychiatry. Biological Psychiatry, 31, 1079-1081.

Gabbard, G. O. (1992) Psychodynamic psychiatry in the 'decade of the brain'. American Journal of Psychiatry, 149. 991-998.

HofstaDTER, D. R. (1981) Reflections. In The Minds I. Fantasies and Reflections on Self and Soul (eds D. R. Hofstadter \& D. C. Dennett). New York: Bantam Books.

Hudson, J. I. \& POPE, JR. H. G. (1990) Affective spectrum disorder: Does antidepressant response identify a family of disorders with a common pathophysiology? American Journal of Psychiatry, 147, 552-564.

HuNDERT, E. M. (1989) Philosophy, Psychiatry and Neuroscience. Three Approaches to the Mind. Oxford: Clarendon Press.

MaAs, J. W. \& KATZ, M. M. (1992) Neurobiology and psychopathological states: Are we looking in the right place? Biological Psychiatry, 31, 757-758.

Mandell, A. J. \& Selz, K. A. (1992) Dynamical systems in psychiatry: now what? Biological Psychiatry, 32, 299-301.

MoHL, P. C. (1987) Should psychotherapy be considered a biological treatment? Psychosomatics, 28, 320-326.

PAM, A. (1990) A critique of the scientific status of biological psychiatry. Acta Psychiatrica Scandinavica, 82 (suppl. 362). 
PArdes, H. (1986) Neuroscience and psychiatry: Marriage or coexistence. American Journal of Psychiatry, 143, 1205-1212.

Pardo, J. V., PArdo, P. J. \& Raichle, M. E. (1993) Neural correlates of self-induced dysphoria. American Journal of Psychiatry, 150, 713-719.

van Pranc, H. M. (1989) Moving ahead yet falling behind. A critical appraisal of some trends in contemporary depression research. Neuropsychobiology, 22, 181-193.

Reıser, M. F. (1984) Mind, Brain, Body. Toward a Convergence of Psychoanalysis and Neurobiology. New York: Basic Books.

RuDNICK, A. (1990) Towards rationalization of biological psychiatry: a study in psychobiological epistemology. Journal of Medical Philosophy, 15, 75-96.

SCBNTACOTHA, J. (1993) Self-organization: The basic principle of neural functions. Theoretical Medicine, 14, 101-116.

SPERrY, R. W. (1980) Mind-brain interaction: Mentalism, yes; dualism, no. Neuroscience, 5, 195-206.

Spibozl, D., Kraemer, H. C., Bloom, J. R., et al (1989) Effect of psychosocial treatment on survival of patients with metastatic breast cancer. Lancet, 2, 888-891.

Volkow, N. D. \&. TANCRBDi, L. R. (1991) Biological correlates of mental activity studied with PET. American Journal of Psychiatry, 148, 439-443.

Hasse Karlsson, MD, University of Turku, Department of Psychiatry; Matti Kamppinen, PhD, University of Turku, Department of Cultural Studies

Correspondence: Hasse Karlsson, Louhimontie S, 20540 Turku, Finland

(First received 4 January 1995, final revision 28 February 1995, accepted 18 April 1995) 